\title{
Cuantificación y caracterización de la exclusión residencial en las capitales de la CAPV
}

\section{SIIS Centro de Documentación y Estudios}

Fundación Eguía-Careaga

<estudios@siis.net>

Artikulu honetan biltzen dira aldi berean EAEko hiru hiriburuetan 2012ko Urrian egindako bizitegibazterketa larriko egoerak pairatzen dituzten pertsonen zenbaketaren ondorioak. Zenbaketa hori sustatu zuten Bilbo, Donostia eta Gasteizko udalek, eta lankide izan zituzten bai hiru aldundiak bai Eusko Jaurlaritzaren Enplegu eta Gizarte Politiketako Saila. Jasotako datuen arabera, zenbaketa egindako gau hartan guztira lokalizatu zituzten 243 pertsona kalean lo egiten, eta 1.400 pertsona inguru zenbatu zituzten gizarte-zerbitzuetako sarearen ostatu hartzeko zentroetan.

\section{HITZ-GAKOAK:}

Gizarte-bazterketa, etxegabekoak, ezaugarriak, detekzioa, Euskadi.
Este artículo recoge las principales conclusiones del recuento de personas en situación de exclusión residencial extrema realizado de forma simultánea en las tres capitales de la CAPV, en octubre de 2012, con el objetivo de cuantificar y caracterizar a las personas en situación de exclusión residencial extrema que residen en ellas. El recuento fue promovido por los ayuntamientos de Bilbao, Donostia-San Sebastián y Vitoria-Gasteiz, y colaboraron en su realización tanto las tres diputaciones forales como el Departamento de Empleo y Políticas Sociales del Gobierno Vasco. De acuerdo a los datos recogidos, en la noche de recuento fueron localizadas un total de $\mathbf{2 4 3}$ personas pernoctando en la calle, mientras que algo más de 1.400 fueron contabilizadas en los diversos centros de alojamiento de la red de servicios sociales.

\section{Palabras Clave:}

Exclusión social, sin techo, características, detección, País Vasco. 


\section{Presentación: antecedentes, objetivos y metodología}

Este artículo recoge los principales resultados de la investigación realizada de forma simultánea en las tres capitales de la CAPV, en octubre de 2012, con el objetivo de cuantificar y caracterizar a las personas en situación de exclusión residencial extrema que residen en ellas ${ }^{1}$. El trabajo se basa en la experiencia de las denominadas 'noches $S$ ' -el término en castellano se deriva del inglés shelter and streetnight- o recuentos censales nocturnos, realizados de forma cada vez más habitual en ciudades de nuestro entorno.

Los objetivos de la investigación realizada han sido los siguientes:

- Cuantificar el número de personas en situación de exclusión residencial grave en el marco de la CAPV, tomando como referencia la noche del 17 al 18 de octubre de 2012, considerando tanto las personas que durmieron en calle en las tres capitales vascas como aquellas que estuvieron alojadas durante la mencionada noche en los diferentes centros y servicios con alojamiento dispuestos en los tres territorios históricos para atender a las personas en situación de exclusión social.

- Analizar la situación de estas personas en aspectos relacionados con su situación administrativa, nivel educativo, actividad e ingresos, trayectoria hacia la exclusión residencial, estado de salud, consumo de drogas, relaciones familiares y sociales, y uso, demanda y valoración de servicios sociales.

- Conocer las características sociodemográficas más importantes de las personas localizadas, identificar la prevalencia de determinados indicadores críticos en cuanto a su situación y analizar su incidencia según determinadas características básicas, como la edad, el sexo, la nacionalidad, el lugar de ubicación o la situación residencial durante la noche del recuento.

- Obtener una imagen general del mapa de recursos con alojamiento existente en la CAPV para atender las necesidades de las personas en condiciones de exclusión social, ofreciendo un acercamiento preliminar a la distribución de estos recursos según las categorías de servicios establecidas en el catálogo de la Ley 12/2008, de 5 de diciembre, de Servicios Sociales.

Desde el punto de vista metodológico, el estudio se basa, al igual que los trabajos realizados previamente en Bilbao, en 2010, y en Donostia-San Sebastián, en 2011, en el marco conceptual relativo a las personas en situación de exclusión desarrollado por Feantsa en el Observatorio Europeo sobre las Personas sin Hogar (2005). La definición de la tipo-

${ }^{1}$ El informe completo del estudio puede consultarse en la página web del SIIS Centro de Documentación y Estudios, así como en las sedes electrónicas de los ayuntamientos que han participado en esta iniciativa (〈http://www.siis.net/es/ver-detalle.php?ref=211784)). logía ETHOS parte de la base de que un hogar puede estar constituido por tres espacios o 'dominios' (físico, social y legal), de manera que la ausencia en mayor o menor grado de alguno de ellos configura las distintas formas de sinhogarismo que pueden darse. De este modo, de la combinación de estos tres dominios se desprenden cuatro categorías ordinales (en la medida en que se distribuyen desde un menor grado de exclusión a uno mayor), que constituyen las cuatro categorías básicas de la tipología europea del sinhogarismo y la exclusión residencial ETHOS:

\section{- Sin techo.}

- Sin vivienda

- Vivienda insegura.

- Vivienda inadecuada.

A partir de estas cuatro categorías conceptuales, se desprenden trece categorías operativas, que varían a lo largo de continuo en el que se contemplan desde la situación de aquellas personas que se encuentran literalmente en la calle y utilizan espacios públicos para dormir, hasta la de aquellas otras que sí disponen de un techo para dormir, pero se alojan en viviendas en situación de hacinamiento extremo. Por otra parte, este marco conceptual permite también clasificar a las personas en situación de exclusión residencial según las condiciones que presenta el lugar en el que habitan, y no en términos de sus rasgos personales. Debe precisarse, sin embargo, que esta tipología contempla el sinhogarismo en sentido muy amplio y que la presente investigación no hace referencia a todas las situaciones de exclusión residencial que se recogen en ella. Obviamente, hubiera sido deseable la incorporación de todas las situaciones recogidas en el marco conceptual propuesto por ETHOS - lo que, sin duda, nos habría hecho considerar la exclusión residencial como un problema de mucho mayor alcance-; sin embargo, ni las fuentes estadísticas disponibles ni el alcance limitado de la presente investigación lo han hecho posible.

Así pues, y como se observa en la tabla, del conjunto de las categorías operativas y situaciones residenciales recogidas en la tipología ETHOS, la investigación realizada se ha centrado fundamentalmente en las englobadas bajo las categorías conceptuales de 'sin techo' y 'sin vivienda'. El ámbito poblacional objeto de estudio de esta investigación lo componen, por tanto: a) personas sin hogar, de cualquier edad, que duermen en la calle, bien a la intemperie, en un espacio de uso público a cubierto o en cualquier otro lugar inadecuado que pueda ser observado desde la calle; y b) personas sin hogar, de cualquier edad, que se alojan en alguno de los centros o servicios con alojamiento que se dirigen a atender a estas personas.

Como se ha señalado previamente, el ámbito geográfico del estudio se centra específicamente en las ciudades de Donostia-San Sebastián, Bilbao y VitoriaGasteiz, si bien también se ofrece información respecto a personas residentes en recursos de alojamiento ubicados en otros 23 municipios de Bizkaia y Gipuzkoa. 
Tabla 1. Tipos de situaciones de exclusión residencial que abarca la presente investigación, de acuerdo con la tipología ETHOS

\begin{tabular}{|c|c|c|c|c|c|}
\hline $\begin{array}{l}\text { Categoría } \\
\text { conceptual }\end{array}$ & \multicolumn{2}{|c|}{ Categoría operativa } & \multicolumn{2}{|c|}{ Situación residencial } & \multirow{2}{*}{$\begin{array}{c}\text { Investigación } \\
\text { Sí }\end{array}$} \\
\hline \multirow[b]{2}{*}{ Sin techo } & 1 & $\begin{array}{l}\text { Personas que viven en un espacio público } \\
\text { (a la intemperie) }\end{array}$ & 1.1 & Espacio público y exterior & \\
\hline & 2 & $\begin{array}{l}\text { Personas que duermen en un refugio } \\
\text { nocturno o se ven obligadas a pasar varias } \\
\text { horas al día en un espacio público }\end{array}$ & 2.1 & Albergue o refugio nocturno & Sí \\
\hline \multirow{11}{*}{ Sin vivienda } & \multirow{3}{*}{3} & \multirow{3}{*}{$\begin{array}{l}\text { Personas que viven en albergues y centros } \\
\text { para gente sin hogar o en alojamientos } \\
\text { temporales }\end{array}$} & 3.1 & Albergues y centros de alojamiento & Sí \\
\hline & & & 3.2 & Alojamiento temporal y de tránsito & Sí \\
\hline & & & 3.3 & Alojamiento con apoyo & Sí \\
\hline & 4 & Personas en albergues para mujeres & 4.1 & $\begin{array}{l}\text { Albergues para mujeres (solas o con } \\
\text { hijos) }\end{array}$ & Sí \\
\hline & \multirow{2}{*}{5} & \multirow{2}{*}{$\begin{array}{l}\text { Personas en centros de alojamiento para } \\
\text { solicitantes de asilo e inmigrantes }\end{array}$} & 5.1 & $\begin{array}{l}\text { Alojamiento temporal / centros de } \\
\text { recepción }\end{array}$ & Sí \\
\hline & & & 5.2 & $\begin{array}{l}\text { Alojamientos para trabajadores } \\
\text { temporeros }\end{array}$ & No \\
\hline & \multirow{3}{*}{6} & \multirow{3}{*}{$\begin{array}{l}\text { Personas que en un plazo definido van a ser } \\
\text { despedidas de instituciones residenciales o } \\
\text { de internamiento }\end{array}$} & 6.1 & Instituciones penales (cárceles) & No \\
\hline & & & 6.2 & Instituciones sanitarias (hospitales) & Sí (sólo en Bizkaia) \\
\hline & & & 6.3 & Centros de menores & Sí (sólo en Bizkaia) \\
\hline & \multirow[b]{2}{*}{7} & \multirow{2}{*}{$\begin{array}{l}\text { Personas que reciben alojamiento con } \\
\text { apoyo sostenido, debido a su condición de } \\
\text { personas sin hogar }\end{array}$} & 7.1 & $\begin{array}{l}\text { Residencia para personas sin hogar } \\
\text { mayores }\end{array}$ & No \\
\hline & & & 7.2 & $\begin{array}{l}\text { Vivienda tutelada y con apoyo a largo } \\
\text { plazo para personas anteriormente } \\
\text { sin hogar }\end{array}$ & Sí \\
\hline \multirow{6}{*}{$\begin{array}{l}\text { Vivienda } \\
\text { insegura }\end{array}$} & \multirow{3}{*}{8} & \multirow{3}{*}{$\begin{array}{l}\text { Personas que viven en un régimen de } \\
\text { tenencia inseguro o sin pagar alquiler }\end{array}$} & 8.1 & Acogidas por familiares & No \\
\hline & & & 8.2 & $\begin{array}{l}\text { Sin tenencia legal (en régimen de } \\
\text { subarriendo, por ejemplo) }\end{array}$ & No \\
\hline & & & 8.3 & Ocupación ilegal & No \\
\hline & \multirow{2}{*}{9} & \multirow{2}{*}{$\begin{array}{l}\text { Personas que viven bajo amenaza de } \\
\text { desahucio }\end{array}$} & 9.1 & En régimen de alquiler & No \\
\hline & & & 9.2 & Con la vivienda en propiedad & No \\
\hline & 10 & $\begin{array}{l}\text { Personas que viven bajo amenazas de } \\
\text { violencia por parte de la pareja o de la } \\
\text { familia }\end{array}$ & 10.1 & $\begin{array}{l}\text { Con denuncias presentadas ante la } \\
\text { Policía }\end{array}$ & No \\
\hline \multirow{5}{*}{$\begin{array}{l}\text { Vivienda } \\
\text { inadecuada }\end{array}$} & \multirow{3}{*}{11} & \multirow{3}{*}{$\begin{array}{l}\text { Personas que viven en estructuras } \\
\text { temporales y no convencionales }\end{array}$} & 11.1 & Caravanas y similares & Sí \\
\hline & & & 11.2 & $\begin{array}{l}\text { Edificaciones no convencionales ni } \\
\text { pensadas para que residan personas }\end{array}$ & Sí \\
\hline & & & 11.3 & Estructuras temporales & Sí \\
\hline & 12 & Alojamiento impropio & 12.1 & $\begin{array}{l}\text { Edificio ocupado que no es apropiado } \\
\text { para vivir en él }\end{array}$ & Sí \\
\hline & 13 & Hacinamiento extremo & 13.1 & $\begin{array}{l}\text { Muy por encima de los estándares } \\
\text { habituales que marcan el hacinamiento }\end{array}$ & No \\
\hline
\end{tabular}

Fuente: Elaboración propia a partir de Feantsa (2005).

El trabajo de campo de esta investigación se realizó, de forma simultánea en los tres territorios históricos, entre los días 17 y 18 de octubre de 2012. Su organización fue liderada por los ayuntamientos de Bilbao, Vitoria-Gasteiz y el Grupo de Trabajo Kale Gorrian (del que forman parte el Ayuntamiento de DonostiaSan Sebastián, la Diputación Foral de Gipuzkoa y diversas entidades sociales) y su realización corrió a cargo de un total de 678 personas voluntarias en toda la CAPV. Se contó también con la colaboración de aproximadamente 170 profesionales que desempeñan su trabajo en el resto de centros y servicios con alojamiento, tanto en lo que respecta al recuento de personas alojadas como a la realización de entrevistas personales.

En total, de las 678 personas que participaron como voluntarias en este estudio -dejando al margen a quienes colaboraron como profesionales-, la mayor parte fueron personas voluntarias de las entidades del tercer sector que disponen de programas y servicios en el ámbito de la exclusión social (el 41,3\%) y personas que participaron en la investigación como consecuencia de la colaboración que se produjo con diversos centros educativos, universitarios y colegios profesionales (el 38,3\%). Por otra parte, un 12,7\% de todas ellas eran personas empleadas en el sector público -fundamentalmente procedentes de los respectivos departamentos de servicios sociales de los ayuntamientos de Bilbao, Donostia-San Sebastián y Vitoria-Gasteiz-, mientras que un $7,7 \%$ lo hizo a título individual. El 39,4\% de todas las personas que participaron como voluntarias en esta investigación procedían de Bizkaia; el 37,9\%, de Álava; y el 22,7\% restante, de Gipuzkoa.

Con el fin obtener de forma sistemática y ordenada toda la información necesaria para la realizar esta 
investigación, se utilizaron los siguientes instrumentos comunes de recogida de datos:

- Cuestionario dirigido a personas localizadas en calle y ficha de observación.

- Formulario de indicios para el recuento en calle.

- Cuestionario dirigido a personas usuarias de recursos con alojamiento y ficha de observación.

- Formulario de recogida de datos en centros y servicios con alojamiento.

- Formulario de recogida de datos en centros y servicios de día.

- Ficha de inscripción de las personas voluntarias.

- Ficha de evaluación de la recogida de datos.

Cabe señalar además que, si bien buena parte de estos instrumentos están basados en las mismas herramientas que se utilizaron en los estudios previos de Bilbao (2010) y Donostia-San Sebastián (2011) -y éstos, a su vez, en los cuestionarios utilizados en los recuentos que se hicieron con anterioridad en las ciudades de Madrid y Barcelona-, se ha hecho un esfuerzo importante por mejorar dichas herramientas y dotarlas de una mayor sistematicidad. La tasa de respuesta obtenida fue del $82 \%$ para el conjunto de las personas que constituyen el universo de la investigación, con una tasa algo más elevada entre las personas usuarias de recursos (el $85 \%$ ) que entre las localizadas en la calle (el $60 \%$ en relación al conjunto de esas personas, el $73 \%$ si nos centramos en las que estaban en ese momento despiertas).

A la hora de interpretar los resultados obtenidos en el recuento nocturno de personas $y$, de forma específica, en el realizado en calle, es preciso considerar que este tipo de investigaciones proporcionan una imagen fija e instantánea de este fenómeno, a través de un recuento nocturno de una noche concreta y con un horario limitado. Adicionalmente, debe señalarse que, en esta metodología, pueden incidir determinadas situaciones que conlleven un determinado nivel de error. Para neutralizar estas limitaciones, y como se señala más adelante, se han desarrollado una serie de mediciones alternativas, con las que se pretende ofrecer una estimación del número personas que efectivamente pernoctaron en la noche del recuento en las tres capitales vascas.

\section{Recuento de las personas en situación de exclusión residencial grave en la CAPV}

\subsection{Las personas localizadas pernoctando en la calle}

Durante la noche del recuento fueron localizadas, en las tres capitales de la CAPV, 243 personas pernoctando en espacios públicos a la intemperie o en lugares inadecuados (calles y plazas; túneles u otras infraestructuras; coches, caravanas y similares; cajeros automáticos; edificios abandonados o chabolas; entre otros). En la ciudad de Bilbao, fueron localizadas 148 (el 60,9\%); en Donostia-San Sebastián 66
( $27,2 \%) ;$ y en Vitoria-Gasteiz 29 (el 11,9\%). De las 243 personas localizadas, 45 estaban dormidas y 198 despiertas.

Gráfico 1. Resultados del recuento de personas en calle. Bilbao, Donostia-San Sebastián y Vitoria-Gasteiz, noche del 17 al 18 de octubre de 2012

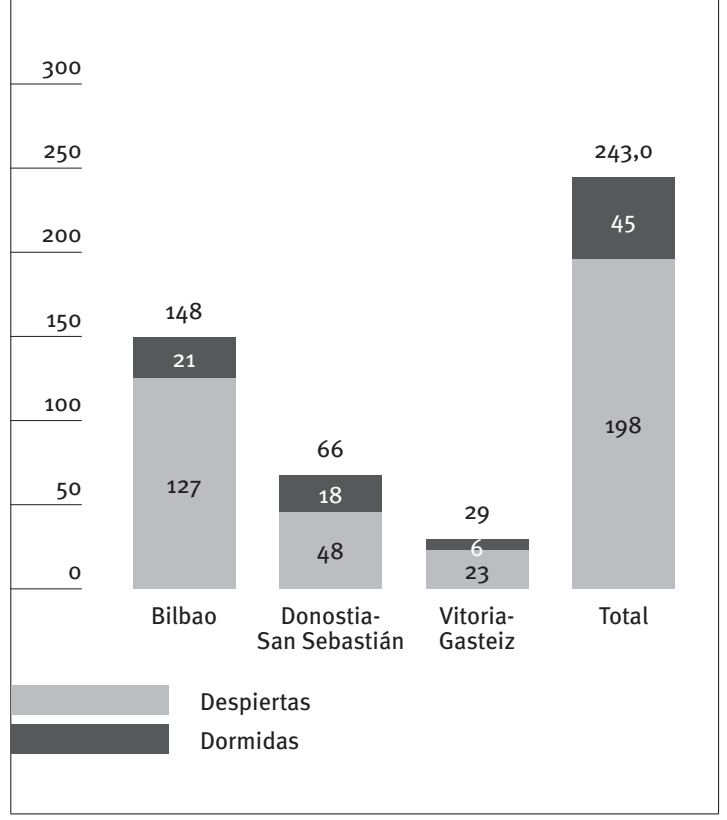

Fuente: Elaboración propia.

En cualquier caso, la cifra de 243 personas debe apreciarse como la correspondiente al número de personas sin hogar en calle que, efectivamente, fueron vistas y sobre las que se dispone de información precisa al respecto. Sin embargo, ello no quiere decir, ni mucho menos, que se haya sido capaz de llegar a todas las personas a las que se pretendía haber llegado. Las mediciones alternativas realizadas mediante otros dos sistemas complementarios de estimación sugieren que esta cifra podría incrementarse con entre 119 y 145 personas más, de forma que, en relación al número de personas que efectivamente fueron localizadas en calle, la cifra real de personas sin hogar podría ascender hasta en un $61,3 \%$. La ciudad que, utilizando los dos métodos de recuento complementarios, más vería aumentado el número de personas sin hogar es Vitoria-Gasteiz (el $72 \%$ ); y la que menos, Donostia-San Sebastián (el $56 \%$ ). Bilbao se situaría entre las dos, con un incremento del $61,5 \%$.

En lo que se refiere a la distribución geográfica de las personas localizadas, cabe señalar que, en Bilbao, la mayor proporción de personas localizadas (el 20,9\%) correspondió al distrito de Deusto; en Donostia-San Sebastián, el mayor número de personas localizadas se encontró en la zona de Amara Berri-Loiola-Martutene (39,4\%); mientras que en Vitoria-Gasteiz, la mayor proporción correspondió al Distrito 3 (Aranbizkarra, Santiago y Arana, fundamentalmente), con el 37,9\% de todas las personas detectadas. 
Además de considerar la distribución geográfica de las personas localizadas durante la noche del recuento, resulta de gran interés analizar el tipo de ubicación en la que se encontraban. En este sentido, existen dos datos fundamentales que deben tenerse en cuenta: por una parte, el lugar en el que las personas contabilizadas fueron localizadas; y, por otra, el lugar de pernocta, no siempre coincidente con el primero. Esta diferenciación resulta pertinente en la medida en que no todas las personas localizadas -dejando al margen, como es lógico, las que estaban dormidas- señalaron que fueran a dormir en el mismo lugar en el que se las localizó. En efecto, tal y como puede observarse en la Tabla 2, el $16 \%$ de todas las personas contabilizadas (es decir, 39 personas) manifestaron que esa noche dormirían en la calle, pero en un lugar distinto al de localización.

Tabla 2. Resultados del recuento de personas en calle, según su ubicación y el lugar de pernocta

\begin{tabular}{|c|c|c|c|c|c|}
\hline & \multirow[b]{2}{*}{ Dormidas } & \multicolumn{3}{|c|}{ Despiertas } & \multirow[b]{2}{*}{ Tota } \\
\hline & & $\begin{array}{l}\text { Durmieron } \\
\text { en el lugar } \\
\text { donde fueron } \\
\text { localizadas }\end{array}$ & $\begin{array}{c}\text { Durmieron } \\
\text { en un lugar } \\
\text { distinto } \\
\text { al de } \\
\text { localización }\end{array}$ & Total & \\
\hline Bilbao & 21 & 105 & 22 & 127 & 148 \\
\hline $\begin{array}{l}\text { Donostia- } \\
\text { San } \\
\text { Sebastián }\end{array}$ & 18 & 33 & 15 & 48 & 66 \\
\hline $\begin{array}{l}\text { Vitoria- } \\
\text { Gasteiz }\end{array}$ & 6 & 21 & 2 & 23 & 29 \\
\hline Total & 45 & 45 & 45 & 45 & 45 \\
\hline
\end{tabular}

Fuente: Elaboración propia.
Considerando las tres ciudades del estudio conjuntamente, cabe decir que más de un tercio de las personas sin hogar $(35,4 \%)$ fueron localizadas en calles, plazas o parques. A estos lugares les siguen, en frecuencia, las infraestructuras y sus aledaños $(18,1 \%)$, los lugares cubiertos en el interior o en las proximidades de edificios públicos o particulares (11,1\%), y las casas o edificios abandonados (9,5\%). El panorama es algo distinto si nos fijamos en cada una de las capitales. El lugar más habitual de las localizaciones coincide con el de la fotografía general, si bien Bilbao reúne el 30,4\% de los casos, y Donostia-San Sebastián y Vitoria-Gasteiz, alrededor del $40 \%$. En términos relativos, en Bilbao se podría destacar el alto porcentaje de personas localizadas en casas o edificios abandonados (12,8\%); en Donostia-San Sebastián, las encontradas en chabolas ( $9,1 \%)$; y en Vitoria-Gasteiz, en vehículos $(20,7 \%)$ y lugares no previstos para vivir (otro 20,7\%).

En cuanto al lugar de pernocta, la fotografía es idéntica en Vitoria-Gasteiz, mientras que cambia un poco en Bilbao y Donostia-San Sebastián. El 12,9\% de las personas sin hogar entrevistadas en calle en Bilbao dijeron que dormirían en un lugar distinto a aquel donde se encontraban en el momento de la entrevista, porcentaje que, en Donostia-San Sebastián, asciende al $28,8 \%$. En la mayor parte de los casos (68,4\% y $47,4 \%$, respectivamente), se trata de personas que fueron localizadas en calles, plazas o parques, pero se desplazaron a otro sitio -seguramente más resguardado- donde dormir. Estas cifras permiten hacerse una idea -en todo caso, muy aproximada- de las pautas de movilidad nocturna de las personas sin hogar que no duermen en centros de alojamiento.

Tabla 3. Resultados del recuento de personas, según el lugar de localización

\begin{tabular}{l|c|c|c|c|c|c|c|c}
\cline { 2 - 9 } & \multicolumn{2}{|c}{ Bilbao } & \multicolumn{2}{c|}{$\begin{array}{c}\text { Donostia- } \\
\text { San Sebastián }\end{array}$} & \multicolumn{2}{c|}{ Vitoria-Gasteiz } & \multicolumn{2}{c}{ Total } \\
\cline { 2 - 10 } & Abs. & $\%$ & Abs. & $\%$ & Abs. & $\%$ & Abs. & $\%$ \\
\hline En la calle o en una plaza & 32 & 21,6 & 25 & 37,9 & 7 & 24,1 & 64 & 26,3 \\
\hline $\begin{array}{l}\text { En un parque } \\
\text { o particulares (a cubierto) }\end{array}$ & 13 & 8,8 & 4 & 6,1 & 5 & 17,2 & 22 & 9,1 \\
\hline $\begin{array}{l}\text { En el interior o alrededores de infraestructuras } \\
\text { (puentes, túneles) }\end{array}$ & 41 & 27,7 & 1 & 1,5 & 2 & 6,9 & 44 & 18,1 \\
\hline En una casa o edificio abandonado & 19 & 12,8 & 4 & 6,1 & 0 & 0,0 & 23 & 9,5 \\
\hline Dentro de un vehículo & 0 & 0,0 & 3 & 4,5 & 6 & 20,7 & 9 & 3,7 \\
\hline En un lugar no previsto para vivir & 5 & 3,4 & 1 & 1,5 & 6 & 20,7 & 12 & 4,9 \\
\hline En un cajero automático & 9 & 6,1 & 4 & 6,1 & 1 & 3,4 & 14 & 5,8 \\
\hline En un establecimiento comercial & 4 & 2,7 & 2 & 3,0 & 0 & 0,0 & 6 & 2,5 \\
\hline En una chabola & 6 & 4,1 & 6 & 9,1 & 1 & 3,4 & 13 & 5,3 \\
\hline En una tienda de campaña & 4 & 2,7 & 2 & 3,0 & 1 & 3,4 & 7 & 2,9 \\
\hline En la playa & 0 & 0,0 & 2 & 3,0 & 0 & 0,0 & 2 & 0,8 \\
\hline Total & $\mathbf{1 4 8}$ & $\mathbf{1 0 0 , 0}$ & $\mathbf{6 6}$ & $\mathbf{1 0 0 , 0}$ & $\mathbf{2 9}$ & $\mathbf{1 0 0 , 0}$ & $\mathbf{2 4 3}$ & $\mathbf{1 0 0 , 0}$ \\
\hline
\end{tabular}

Fuente: Elaboración propia. 
Tabla 4. Resultados del recuento de personas en calle, según el lugar de pernocta

\begin{tabular}{|c|c|c|c|c|c|c|c|c|}
\hline & \multicolumn{2}{|c|}{ Bilbao } & \multicolumn{2}{|c|}{$\begin{array}{c}\text { Donostia- } \\
\text { San Sebastián }\end{array}$} & \multicolumn{2}{|c|}{ Vitoria-Gasteiz } & \multicolumn{2}{|c|}{ Total } \\
\hline & Abs. & $\%$ & Abs. & $\%$ & Abs. & $\%$ & Abs. & $\%$ \\
\hline En la calle o en una plaza & 23 & 15,5 & 16 & 24,2 & 7 & 24,1 & 46 & 18,9 \\
\hline En un parque & 9 & 6,1 & 4 & 6,1 & 5 & 17,2 & 18 & 7,4 \\
\hline En el bosque/monte & 1 & 0,7 & 1 & 1,5 & 0 & 0,0 & 2 & 0,8 \\
\hline $\begin{array}{l}\text { En el interior o alrededores de edificios públicos } \\
\text { o particulares (a cubierto) }\end{array}$ & 14 & 9,5 & 17 & 25,8 & 0 & 0,0 & 31 & 12,8 \\
\hline $\begin{array}{l}\text { En el interior o alrededores de infraestructuras } \\
\text { (puentes, túneles) }\end{array}$ & 42 & 28,4 & 0 & 0,0 & 2 & 6,9 & 44 & 18,1 \\
\hline En una casa o edificio abandonado & 22 & 14,9 & 4 & 6,1 & 0 & 0,0 & 26 & 10,7 \\
\hline Dentro de un vehículo & 0 & 0,0 & 3 & 4,5 & 6 & 20,7 & 9 & 3,7 \\
\hline En lugar no previsto para vivir & 5 & 3,4 & 1 & 1,5 & 6 & 20,7 & 12 & 4,9 \\
\hline En un cajero automático & 9 & 6,1 & 6 & 9,1 & 1 & 3,4 & 16 & 6,6 \\
\hline En un establecimiento comercial & 4 & 2,7 & 3 & 4,5 & o & 0,0 & 7 & 2,9 \\
\hline En una chabola & 7 & 4,7 & 6 & 9,1 & 1 & 3,4 & 14 & 5,8 \\
\hline En una tienda de campaña & 4 & 2,7 & 2 & 3,0 & 1 & 3,4 & 7 & 2,9 \\
\hline En la playa & o & 0,0 & 2 & 3,0 & 0 & 0,0 & 2 & 0,8 \\
\hline Sin datos & 8 & 5,4 & 1 & 1,5 & 0 & 0,0 & 9 & 3,7 \\
\hline Total & 148 & 100,0 & 66 & 100,0 & 29 & 100,0 & 243 & 100,0 \\
\hline
\end{tabular}

Fuente: Elaboración propia.

\subsection{Las personas localizadas en los recursos de alojamiento}

En lo que se refiere a los centros o servicios con alojamiento, durante la noche del 17 al 18 de octubre de 2012 se alojaron en ellos un total de 1.461 personas. En términos muy generales, estos datos implican una media de 5,9 personas por recurso y una tasa de ocupación de prácticamente el $77 \%$. Del total de personas contabilizadas, 616 (el 42,1\%) se encontraban en Bilbao, y el 19,1\% (279 personas) y el $19 \%$ (272 personas), respectivamente, en Vitoria-Gasteiz y Donostia-San Sebastián. El resto de personas permanecieron alojadas en otros municipios guipuzcoanos (el $11,4 \%$ ) o vizcaínos (8,4\%).

Tabla 5. Centros participantes en el estudio, número de plazas y recuento de personas alojadas en recursos con alojamiento

\begin{tabular}{l|c|c|c|c}
\cline { 2 - 5 } & Álava & Bizkaia & Gipuzkoa & CAPV \\
\hline $\begin{array}{l}\text { Centros } \\
\text { participantes }\end{array}$ & 41 & 140 & 68 & 249 \\
\hline Número de plazas & 406 & 921 & 576 & 1.903 \\
\hline $\begin{array}{l}\text { Personas } \\
\text { alojadas }\end{array}$ & 279 & 738 & 444 & 1.461 \\
\hline $\begin{array}{l}\text { Media de plazas } \\
\text { por recurso }\end{array}$ & 9,9 & 6,6 & 8,5 & 7,6 \\
\hline $\begin{array}{l}\text { Media de } \\
\text { personas por } \\
\text { recurso }\end{array}$ & 6,8 & 5,3 & 6,5 & 5,9 \\
\hline $\begin{array}{l}\text { Tasa de } \\
\text { ocupación (\%) }\end{array}$ & 68,7 & 80,1 & 77,1 & 76,8 \\
\hline
\end{tabular}

Fuente: Elaboración propia.
A este recuento habría que añadir además la cifra de 37 personas alojadas en comunidades terapéuticas (15 personas), hospitales de psiquiatría (10 personas) y centros de menores (12 personas) que se disponían a abandonar próximamente esos centros y no contaban con un hogar al que acudir, lo que situaría el recuento en 1.498 personas. Esta información tan sólo se refiere a Bizkaia; con todo, se ha optado por incluirla en el análisis, si bien exclusivamente en las tablas de recuento de ETHOS.

Los centros que concentraron a un mayor número de personas fueron los albergues municipales de las tres capitales vascas. El albergue municipal de Elejabarri (Bilbao) alojó durante la noche del recuento a 71 personas, y los centros municipales de acogida social (CMAS) de Vitoria-Gasteiz y Donostia-San Sebastián, a 60 y 37 personas, respectivamente. En total, estos tres centros, que suponen el 1,2\% de los existentes, proporcionaron alojamiento a 168 personas, es decir, a un $11,5 \%$ del total de las atendidas esa noche. La distribución de las personas contabilizadas en recursos con alojamiento de acuerdo con la tipología ETHOS pone de manifiesto que casi ocho de cada diez personas, el $77,5 \%$, fueron acogidas en recursos de carácter temporal.

\subsection{Una visión de conjunto}

En conjunto, considerando las personas que fueron localizadas en la calle y a aquellas que durante esa misma noche pernoctaron en recursos con alojamiento, el recuento de la población en situación de exclusión residencial grave comprende 1.741 personas. El $14 \%$ de éstas fueron localizas en calle y el $86 \%$ restante estaban alojadas en diversos 
Tabla 6. Características y número de personas alojadas en los diez recursos con alojamiento que registraron una mayor ocupación

\begin{tabular}{|c|c|c|c|c|c|}
\hline Nombre & Municipio & Titularidad & $\begin{array}{l}\text { Personas } \\
\text { alojadas }\end{array}$ & $\begin{array}{l}\text { \% personas } \\
\text { alojadas sobre } \\
\text { el total }\end{array}$ & $\begin{array}{c}\text { Tasa de } \\
\text { ocupación del } \\
\text { centro }\end{array}$ \\
\hline Albergue Municipal de Elejabarri & Bilbao & Municipal & 71 & 4,9 & 100,0 \\
\hline Centro Municipal de Acogida Social & Vitoria-Gasteiz & Municipal & 60 & 4,1 & 89,6 \\
\hline Centro Municipal de Acogida Social & Donostia-San Sebastián & Municipal & 37 & 2,5 & 92,5 \\
\hline Centro de Acogida de Puente Alto & Vitoria-Gasteiz & Privada & 34 & 2,3 & 97,1 \\
\hline Centro de Noche Lagun Artean & Bilbao & Privada & 31 & 2,1 & 96,9 \\
\hline Gaueko Aterpea & Donostia-San Sebastián & Municipal & 30 & 2,1 & 93,8 \\
\hline Albergue Municipal de Baja Exigencia & Bilbao & Municipal & 28 & 1,9 & 87,5 \\
\hline Lurberri Etxea & Bilbao & Privada & 25 & 1,7 & 89,3 \\
\hline Centro de Noche Aterpe & Vitoria-Gasteiz & Municipal & 23 & 1,6 & 71,9 \\
\hline Pensión Manuel Allende - Giltza & Bilbao & Privada & 22 & 1,5 & 81,5 \\
\hline Total & - & - & 361 & 24,7 & 100,0 \\
\hline
\end{tabular}

Fuente: Elaboración propia.

recursos asistenciales con alojamiento. No obstante, si se consideran exclusivamente las cifras que son comparables para los tres territorios, este recuento se situaría en 1.704 personas, ya que, a diferencia de en Bizkaia, ni en Álava ni en Gipuzkoa se consignó el dato de personas en comunidades terapéuticas, centros de menores y hospitales psiquiátricos. Los datos recogidos ponen de manifiesto que la proporción de personas localizadas en calle sobre el total de las personas contabilizadas es, en Bizkaia, algo mayor $(16,7 \%)$ que en Gipuzkoa (12,9\%) y, sobre todo, que en Álava (9,4\%).

En lo que se refiere a la incidencia de las diversas situaciones de exclusión residencial grave registradas, los resultados obtenidos indican que, en Bilbao, la tasa de incidencia de personas localizadas en calle (0,42 personas por cada 1.000 habitantes) es algo superior a la registrada en Donostia-San Sebastián (o,35 personas por cada 1.000 habitantes) y triplica ampliamente a la correspondiente a la capital alavesa (o,12 personas por cada 1.000 habitantes). Aunque no puede afirmarse con total rotundidad, muy posiblemente estos datos se deban a una prevalencia del sinhogarismo realmente más elevada en Bilbao que en las otras dos capitales.

La situación varía cuando se observa la tasa de incidencia de personas alojadas en recurso. En este caso, mientras que Bizkaia (o,64 personas por cada 1.000 habitantes) y Gipuzkoa (o,62 personas por cada 1.000 habitantes) presentan una incidencia relativamente similar, Álava, con o,86 personas en recurso por cada 1.000 habitantes, se sitúa claramente a la cabeza de los tres territorios. Esta mayor incidencia observada en Álava coincide también con una mayor oferta asistencial de plazas. En efecto, mientras que Bizkaia y Gipuzkoa cuentan con o,8 plazas por cada 1.000 habitantes, en Álava la oferta asistencial de los centros contabilizados en este estudio (1,3 plazas por 1.000 habitantes) es significativamente mayor que la de los otros dos territorios.
Tabla 7. Incidencia y distribución de las diversas situaciones de exclusión residencial grave

\begin{tabular}{l|c|c|c|c}
\cline { 2 - 5 } & Álava & Bizkaia & Gipuzkoa & CAPV \\
\hline $\begin{array}{l}\text { Total personas en calle y } \\
\text { recursos }\end{array}$ & 308 & 308 & 308 & 308 \\
\hline $\begin{array}{l}\text { Personas localizadas } \\
\text { en calle }\end{array}$ & 29 & 148 & 66 & 243 \\
\hline $\begin{array}{l}\text { Personas ubicadas en } \\
\text { recursos con alojamiento }\end{array}$ & 279 & 738 & 444 & 1.461 \\
\hline $\begin{array}{l}\text { Tasa por 1.00o } \\
\text { habitantes de personas } \\
\text { en calle* }\end{array}$ & 0,12 & 0,42 & 0,35 & 0,31 \\
\hline $\begin{array}{l}\text { Tasa por 1.00o } \\
\text { habitantes de personas } \\
\text { en recurso** }\end{array}$ & 0,86 & 0,64 & 0,62 & 0,67 \\
\hline $\begin{array}{l}\text { Tasa por 1.00o } \\
\text { habitantes (total)** }\end{array}$ & 0,95 & 0,76 & 0,72 & 0,78 \\
\hline $\begin{array}{l}\text { \% de personas en calle } \\
\text { sobre el total }\end{array}$ & 9,4 & 16,7 & 12,9 & 14,3 \\
\hline $\begin{array}{l}\text { \% de personas alojadas } \\
\text { en recurso sobre el total }\end{array}$ & 90,6 & 83,3 & 87,1 & 85,7 \\
\hline
\end{tabular}

* Puesto que los datos se refieren exclusivamente a las personas localizadas en las capitales, la tasa se calcula sobre esa población. ** La tasa se calcula sobre el total de personas residentes en cada territorio histórico.

Fuente: Elaboración propia.

\subsection{Comparación con otras realidades y datos de evolución}

Si se compara la incidencia de personas en calle obtenida en este estudio con la de otros similares realizados en Madrid, Barcelona o Zaragoza, los resultados ponen de manifiesto que la tasa de incidencia más alta se observa en la ciudad de Barcelona. En este sentido, los datos del último recuento realizado en esta ciudad en 2011 (838 personas) revelan una incidencia de casi 0,52 personas por cada 1.000 habitantes, superior, por tanto, a la registrada tanto en Bilbao $(0,42)$ y el resto de capitales vascas, como en Zaragoza $(0,23)$ e, incluso, Madrid $(0,22)$. No puede afirmarse, sin embargo, con la suficiente precisión que estas diferencias se deban a una prevalencia del sinhogarismo realmente más elevada, o por 
el contrario, a una mayor capacidad de detección de personas en calle por parte de los equipos encargados del recuento.

Por último, los datos de evolución -respecto a los estudios realizados en 2010 en Bilbao y en 2011 en Donostia-San Sebastián- ponen de relieve que tanto en un territorio como en otro se ha producido un cierto aumento en el número total de personas en situación de exclusión social grave. En el caso de Bizkaia, se ha pasado de 854 personas contabilizadas en el verano de 2010 a 923 en 2012 , lo que supone un incremento del 8,1\%. En Gipuzkoa, este incremento ha sido algo menor $(6,3 \%)$, registrándose en 2012, 29 personas más que el año anterior. Sin embargo, mientras en Bizkaia el incremento se deriva fundamentalmente de un mayor número de personas acogidas en recurso, ya que las personas localizadas en la calle se han reducido en un $\mathbf{2 7} \%$, en DonostiaSan Sebastián el número de personas acogidas en centros se mantiene estable, mientras que se contabilizaban 21 personas más en la calle, lo que supone un incremento del $46 \%$.

Tabla 8. Comparación de los resultados obtenidos en el estudio con los realizados en Bizkaia y Gipuzkoa. 2010/2011-2012

\begin{tabular}{|c|c|c|c|c|c|c|}
\hline & & & & & \\
\hline & & \multicolumn{2}{|c|}{2010} & \multicolumn{2}{|c|}{2012} & $\Delta$ \\
\hline & & Abs. & $\%$ & Abs. & $\%$ & $\%$ \\
\hline \multirow{3}{*}{ Bizkaia } & Calle (Bilbao) & 205 & 24,0 & 148 & 16,0 & $-27,8$ \\
\hline & Recurso & 649 & 76,0 & 775 & 84,0 & 19,4 \\
\hline & Total & 854 & 100,0 & 923 & 100,0 & 8,1 \\
\hline & & \multicolumn{2}{|c|}{2011} & \multicolumn{2}{|c|}{2012} & $\Delta$ \\
\hline & & Abs. & $\%$ & Abs. & $\%$ & $\%$ \\
\hline \multirow{3}{*}{ Gipuzkoa } & \begin{tabular}{|l} 
Calle \\
(Donostia- \\
San \\
Sebastián) \\
\end{tabular} & 45 & 5,3 & 66 & 7,2 & 46,7 \\
\hline & Recurso & 419 & 49,1 & 427 & 46,3 & 1,9 \\
\hline & Total & 464 & 54,3 & 493 & 53,4 & 6,3 \\
\hline
\end{tabular}

Fuente: Elaboración propia a partir de los datos del presente estudio, Deloitte (2010) y SIIS Centro de Documentación y Estudios (2011).

\section{Características de las personas en situación de exclusión residencial grave}

\subsection{Características básicas: sexo, edad, origen y nivel formativo}

Desde el punto de vista de las características del grupo de personas analizado, cabe destacar fundamentalmente el predominio de hombres, personas jóvenes e inmigrantes. En efecto, del total de personas sobre las que se dispone de información, el $82,7 \%$ son hombres, el $46 \%$ tienen menos de 35 años y seis de cada diez (concretamente, el 59,2\%) son de origen extranjero.
Tabla 9. Datos sociodemográficos básicos

\begin{tabular}{l|c|c|c}
\cline { 2 - 4 } & Calle & Alojamiento & Total \\
\hline Hombres (\%) & 93,2 & 81,7 & 82,9 \\
\hline Mujeres (\%) & 6,8 & 18,3 & 17,1 \\
\hline Edad media (años) & 36,9 & 35,6 & 35,7 \\
\hline Tienen menos de 35 años (\%) & 41,1 & 49,6 & 48,7 \\
\hline Tienen más de 54 años (\%) & 11,0 & 8,9 & 9,1 \\
\hline Sin estudios (\%) & 15,1 & 11,1 & 11,6 \\
\hline Han nacido en la CAPV (\%) & 15,1 & 24,9 & 23,8 \\
\hline Han nacido fuera del Estado (\%) & 71,2 & 64,1 & 64,9 \\
\hline Han nacido en el Magreb (\%) & 43,2 & 34,4 & 35,3 \\
\hline
\end{tabular}

Fuente: Elaboración propia.

Desde el punto de vista del lugar de nacimiento, el $35 \%$ de las personas encuestadas han nacido en los países del Magreb (Marruecos y Argelia, fundamentalmente), y el $23 \%$, en la CAPV. El $11 \%$ son personas nacidas en otras comunidades autónomas del Estado y el $12 \%$ provienen de otras zonas diferentes del continente africano. La mitad de las personas extranjeras carece de permiso de residencia. Una de cada diez personas no está empadronada en el Estado español, con lo que no tiene posibilidades de acceso a un buen número de prestaciones y servicios sociales.

En cuanto a su nivel formativo, la mayoría de las personas en exclusión residencial entrevistadas (86,2 \%) cuentan con algún tipo de formación y apenas una de cada diez carece de estudios. Casi la mitad (47,2\%) tienen estudios primarios, y una tercera parte (33,6\%), también secundarios. Cabe señalar además la presencia de un 5,4\% de personas con estudios universitarios.

\subsection{Situación laboral y fuentes de ingresos}

La mayor parte de las personas en situación de exclusión residencial grave no participan en el mercado laboral convencional. Ello no quiere decir que no trabajen, sino que sus actividades, incluso cuando les reportan algo de dinero, no están vinculadas a un empleo o un salario ordinario o convencional. En la encuesta sólo se ha pretendido un acercamiento a estas formas de 'economía informal', cuyo análisis en profundidad requeriría de estudios específicos.

Sólo 55 personas de las 1.313 personas encuestadas, el 4,2\% del total, dicen desempeñar algún trabajo remunerado. El estatus predominante es el del desempleado $(36,7 \%)$ y el de estudiante $(31,3 \%)$, categorías en las que se autoadscriben dos terceras partes del colectivo. Además, hay un $12,3 \%$ de pensionistas y un $14 \%$ de personas en otras situaciones. 
Tabla 10. Distribución de las personas en situación de exclusión residencial grave, según su actividad

\begin{tabular}{l|c|c}
\cline { 2 - 3 } & Abs. & $\begin{array}{c}\text { Distribución } \\
\text { vertical (\%) }\end{array}$ \\
\hline Trabajo & 55 & 4,2 \\
\hline En paro & 482 & 36,7 \\
\hline Jubilado/a, pensionista, & 161 & 12,3 \\
\hline invalidez & 411 & 31,3 \\
\hline Estudia & 184 & 14,0 \\
\hline Otras situaciones & 20 & 1,5 \\
\hline Sin datos & 1.313 & 100,0 \\
\hline Total & &
\end{tabular}

Fuente: Elaboración propia.

Las pocas personas que trabajan lo hacen en ámbitos muy variados, si bien casi la mitad $(45,4 \%)$ se distribuyen en tres sectores: la limpieza ( $20 \%$ ), el cuidado de personas o labores de auxiliar de enfermería $(14,5 \%)$, y la construcción (10,9\%).

Al margen del empleo asalariado convencional, 182 (el $13,9 \%$ de todas las personas en situación de exclusión residencial grave) declaran que desarrollan alguna otra actividad laboral o semilaboral que les reporta ingresos. De ellas, 167 especifican a qué se dedican. Las actividades más frecuentes son, por una parte, el empleo protegido (26,4\%) en talleres ocupacionales, prelaborales o similares; $y$, por otra, otras actividades no reguladas, como la recogida de chatarra $(18,7 \%)$, y el empleo sumergido o la mendicidad $(12,2 \%$, respectivamente).

Tabla 11. Distribución de las personas en situación de exclusión residencial grave que desarrollan otras actividades remuneradas, según actividad o sector de ocupación

\begin{tabular}{|c|c|c|c|}
\hline & Abs. & $\begin{array}{l}\text { Distribución } \\
\text { vertical (\%) }\end{array}$ & $\begin{array}{l}\text { \% sobre } \\
\text { el total }\end{array}$ \\
\hline Empleo protegido & 48 & 26,4 & 3,7 \\
\hline Chatarra & 34 & 18,7 & 2,6 \\
\hline Empleo sumergido & 22 & 12,1 & 1,7 \\
\hline Mendicidad & 22 & 12,1 & 1,7 \\
\hline $\begin{array}{l}\text { Beca de formación / prácticas } \\
\text { remuneradas }\end{array}$ & 12 & 6,6 & 0,9 \\
\hline Venta ambulante & 12 & 6,6 & 0,9 \\
\hline $\begin{array}{l}\text { Ayuda en el negocio de familiares/ } \\
\text { amigos }\end{array}$ & 3 & 1,6 & 0,2 \\
\hline Espectáculos callejeros & 3 & 1,6 & 0,2 \\
\hline Actividades ilegales & 2 & 1,1 & 0,2 \\
\hline Prostitución & 2 & 1,1 & 0,2 \\
\hline Reventa & 2 & 1,1 & 0,2 \\
\hline Vendimia & 2 & 1,1 & 0,2 \\
\hline Trabajos ocasionales & 1 & 0,5 & 0,1 \\
\hline Otros & 2 & 1,1 & 0,2 \\
\hline Sin datos & 15 & 8,2 & 1,1 \\
\hline Total & 182 & 100,0 & 182 \\
\hline
\end{tabular}

Fuente: Elaboración propia.
En lo que se refiere a las fuentes de ingresos de las personas encuestadas, junto al reducido papel de los ingresos laborales, resulta también evidente la escasa cobertura de las prestaciones sociales dirigidas a personas en situación o riesgo de exclusión social, de las que sólo se beneficia una de cada tres personas sin hogar (33\%). La prestación más extendida es la renta de garantía de ingresos (RGI), que, pese a estar diseñada como última malla de seguridad para personas sin recursos o en riesgo de exclusión social, únicamente llega al 18,4\% de las personas identificadas en este estudio. La ayuda de las ONG, recibida por el $14,5 \%$ de las personas sin hogar, se sitúa como tercera fuente de ingresos, justo por debajo de otras prestaciones sociales (14,6\%) y del empleo (11,6\%). La mendicidad sólo es citada como vía de ingresos por el 7,2\%. En cuanto al papel de la ayuda facilitada por familiares, amistades y vecindario, que, en conjunto, alcanza al 11,9\% de las personas encuestadas, merece la pena subrayar que la proporcionada por amigos/as y conocidos/as $(6,4 \%)$ supera a la ofrecida por las familias $(4,9 \%)$, lo cual evidencia una vez más la debilidad del colchón familiar de las personas sin hogar.

Tabla 12. Distribución de las personas en situación de exclusión residencial grave, según fuentes de ingresos (respuesta múltiple)

\begin{tabular}{l|c|c}
\cline { 2 - 3 } & Abs. & \% sobre el total \\
\hline Renta de garantía de ingresos & 241 & 18,4 \\
\hline Otra prestación/ayuda & 192 & 14,6 \\
\hline Ayuda económica de ONG & 191 & 14,5 \\
\hline Trabajo & 152 & 11,6 \\
\hline Pensión & 133 & 10,1 \\
\hline Mendicidad & 95 & 7,2 \\
\hline Ayuda económica de amigo/conocido & 84 & 6,4 \\
\hline Ayuda económica familiar & 64 & 4,9 \\
\hline Prestación/subsidio de desempleo & 62 & 4,7 \\
\hline Ayuda económica de vecino/a & 8 & 0,6 \\
\hline Otros & 87 & 6,6 \\
\hline
\end{tabular}

Fuente: Elaboración propia.

A la pregunta 'En el último mes, si se suman todos sus ingresos, ¿cuánto habría percibido en total, más o menos?', responden el $85,7 \%$ de las personas encuestadas. Tomando en cuenta únicamente las respuestas que señalan algún ingreso (845), la media se situaría en los 392,9 euros mensuales, y la mediana, en 329. Si los cálculos se realizan incluyendo a las personas que no tienen ningún tipo de ingreso, la media quedaría en 295,1 euros mensuales, y la mediana, en 260. Como puede verse, todas estas cifras quedan muy por debajo del salario mínimo interprofesional, que en 2012 estaba fijado en 641,40 euros mensuales. 
Tabla 13. Ingreso medio y mediano de las personas en situación de exclusión residencial grave (euros mensuales)

\begin{tabular}{l|c|c}
\cline { 2 - 3 } & $\begin{array}{c}\text { Ingreso } \\
\text { medio }\end{array}$ & $\begin{array}{c}\text { Ingreso } \\
\text { mediano }\end{array}$ \\
\hline $\begin{array}{l}\text { Considerando todas las respuestas } \\
\text { incluidas las que no tienen ingresos } \\
(N=1.125),\end{array}$ & 295,1 & 260,0 \\
\hline $\begin{array}{l}\text { Considerando sólo las respuestas que } \\
\text { indican algún ingreso }(N=845)\end{array}$ & 392,9 & 329,0 \\
\hline
\end{tabular}

Fuente: Elaboración propia.

¿Son pobres todas las personas en situación de exclusión residencial? Lo son la mayor parte, aunque la cuestión depende en buena medida del indicador que se utilice para determinarlo. De acuerdo con el indicador utilizado en la Encuesta de Pobreza y Desigualdades Sociales (EPDS), realizada por el Gobierno Vasco, el $94,4 \%$ de las personas que han contestado a la pregunta estarían en situación de pobreza en la dimensión de mantenimiento ${ }^{2}$.

Tabla 14. Distribución de las personas en situación de exclusión residencial grave, según su situación de pobreza (indicador de la Encuesta de Pobreza y Desigualdades Sociales)

\begin{tabular}{l|c|c|c}
\cline { 2 - 4 } & Abs. & $\begin{array}{c}\text { Distribución } \\
\text { vertical (\%) }\end{array}$ & $\begin{array}{c}\text { \% sobre las } \\
\text { personas que } \\
\text { responden }\end{array}$ \\
\hline Sí & 1.062 & 80,9 & 94,4 \\
\hline No & 63 & 4,8 & 5,6 \\
\hline Sin datos & 188 & 14,3 & - \\
\hline Total & $\mathbf{1 . 3 1 3}$ & $\mathbf{1 0 0 , 0}$ & $\mathbf{1 0 0 , 0}$ \\
\hline
\end{tabular}

Fuente: Elaboración propia.

Si como referencia se toma el indicador de pobreza grave empleado por Eurostat y por el INE³, cabría considerar en tal situación al 78,2 \% de las personas que responden a la pregunta, no estando, por tanto, un $18 \%$ de estas personas en situación de pobreza severa.

Tabla 15. Distribución de las personas en situación de exclusión residencial grave, según su situación de pobreza (indicador de Eurostat)

\begin{tabular}{l|c|c|c}
\cline { 2 - 4 } & Abs. & $\begin{array}{c}\text { Distribución } \\
\text { vertical (\%) }\end{array}$ & $\begin{array}{c}\% \text { sobre las } \\
\text { personas que } \\
\text { responden }\end{array}$ \\
\hline Sí & 880 & 67,0 & 78,2 \\
\hline No & 245 & 18,7 & 21,8 \\
\hline Sin datos & 188 & 14,3 & - \\
\hline Total & 1.313 & 100,0 & 100,0 \\
\hline
\end{tabular}

Fuente: Elaboración propia.

2 En esta encuesta, el umbral de riesgo de pobreza de mantenimiento para una persona sola menor de 65 años se sitúa en 855 euros.

${ }^{3}$ En este caso, el indicador de pobreza grave para una persona sola se sitúa en 552 euros.
3.3. Condiciones de vida y exclusión residencial: estado de salud, victimización y relaciones sociales

Un tercio (32,7\%) de las personas en situación de exclusión residencial grave identificadas en este estudio llevan en esa situación menos de un año. Cuatro de cada diez personas sin hogar (43,6\%) llevan en situación de exclusión residencial grave entre uno y cinco años, dos de cada diez $(22,2 \%)$ superan ese periodo de tiempo, y un $10 \%$ lleva más de diez años. La razón principal que explica esta situación se relaciona con problemas económicos ( $27,9 \%$ de los casos), seguidos por cuestiones laborales (17,8\%), dificultades en las relaciones familiares o de pareja $(14,4 \%)$, consumo de alcohol u otras drogas (10,3\%) y problemas administrativos (7,4\%). No todas las personas en situación de exclusión residencial han dormido en la calle alguna vez en su vida, pero sí lo ha hecho la mayoría. Siete de cada diez personas encuestadas (72\%) han dormido alguna vez en la calle a lo largo de toda su vida, y la mayoría de las personas que la noche del recuento se encontraban en algún recurso $(68,6 \%)$ ha pasado por esa situación.

Numerosos estudios han puesto de manifiesto en qué medida el hecho de dormir en la calle implica un riesgo importante en relación a la posibilidad de ser víctima de diversos delitos (Newburn y Rock, 2005). Los datos de esta encuesta corroboran claramente esa realidad. Del conjunto de personas encuestadas en la calle y en los centros de alojamiento, más de la mitad $(58,3 \%)$ refieren haber sido víctimas de algún delito o conducta antisocial ligada a esa circunstancia. En cuanto al número de problemas, el 19,2\% menciona sólo uno, y el 39,1\%, más de uno. Los problemas más frecuentes son los insultos y amenazas $(41,5 \%)$, los robos $(39,2 \%)$, las agresiones físicas $(25,4 \%)$ y los timos $(23,3 \%)$.

Tabla 16. Incidencia de diversos problemas sufridos por las personas que han pernoctado alguna vez en calle

\begin{tabular}{|c|c|c|c|}
\hline & Abs. & $\begin{array}{c}\text { \% sobre las que } \\
\text { han dormido en } \\
\text { calle }\end{array}$ & $\begin{array}{c}\% \text { sobre } \\
\text { total }\end{array}$ \\
\hline \multicolumn{4}{|l|}{ Tipo de problema } \\
\hline Agresión física & 240 & 25,4 & 18,3 \\
\hline Robo & 371 & 39,2 & 28,3 \\
\hline Agresión sexual & 26 & 2,7 & 2,0 \\
\hline Timos & 220 & 23,3 & 16,8 \\
\hline Insultos y amenazas & 393 & 41,5 & 29,9 \\
\hline \multicolumn{4}{|l|}{ Número de problemas } \\
\hline Ninguno & 394 & 41,6 & 30,0 \\
\hline Uno & 182 & 19,2 & 13,9 \\
\hline Dos o más & 370 & 39,1 & 28,2 \\
\hline
\end{tabular}

Fuente: Elaboración propia.
En lo que se refiere al estado de salud de las personas encuestadas, si bien ocho de cada diez (85,8\%) personas en situación de exclusión residencial grave 
disponen de tarjeta sanitaria, resulta preocupante que más de una de cada diez (el 13,1\%) carezcan de ese documento, puesto que ello reduce enormemente la asistencia sanitaria pública y gratuita a la que tienen derecho. Por otra parte, más de un tercio de las personas sin hogar que han participado en la encuesta (38,8\%) señalan tener algún problema de salud de tipo grave o crónico. Una cuarta parte de los problemas mencionados se refieren a trastornos o enfermedades mentales ( $25,2 \%$ ), a los que siguen en frecuencia la hepatitis y el $\mathrm{VIH}(14,8 \%)$, y las enfermedades respiratorias (13,3\%). Es importante destacar, por tanto, que de todas las personas entrevistadas, un $15 \%$ han hecho referencia, aunque no necesariamente como problema principal, a los trastornos y las enfermedades mentales, lo que pone de manifiesto el peso de estos problemas en la situación general del colectivo estudiado. Además, conviene destacar que una de cada cinco personas con problemas de salud grave señala no recibir tratamiento sanitario. Se trata, en total, de 104 personas.

En cuanto a las relaciones sociales, la soltería es el estado civil predominante entre las personas en situación de exclusión residencial grave que han respondido al cuestionario, pues caracteriza al $66,9 \%$ de ellas. También hay un $17,2 \%$ de personas separadas o divorciadas y un $9,1 \%$ de personas casadas. Por otra parte, de acuerdo con la información recogida, cuatro de cada cinco $(82,6 \%)$ señalan mantener relaciones con su familia, mientras que el $15,9 \%$ declara lo contrario.

Tabla 17. Distribución de las personas en situación de exclusión residencial grave, según mantengan o no algún contacto familiar

\begin{tabular}{l|c|c}
\cline { 2 - 3 } & Abs. & $\begin{array}{c}\text { Distribución } \\
\text { vertical (\%) }\end{array}$ \\
\hline Mantienen algún contacto & 1.084 & 82,6 \\
\hline No mantienen ningún contacto & 209 & 15,9 \\
\hline Sin datos & 20 & 1,5 \\
\hline Total & $\mathbf{1 . 3 1 3}$ & $\mathbf{1 0 0 , 0}$ \\
\hline
\end{tabular}

Fuente: Elaboración propia.

Finalmente, dos de cada tres personas sin hogar $(69,5 \%)$ pasan la mayor parte del día acompañadas, pero prácticamente tres de cada diez (28,3\%) dicen pasar la mayor parte del día solas.

Tabla 18. Distribución de las personas en situación de exclusión residencial grave, según pasen la mayor parte del día solas 0 acompañadas

\begin{tabular}{l|c|c}
\cline { 2 - 3 } & Abs. & $\begin{array}{c}\text { Distribución } \\
\text { vertical (\%) }\end{array}$ \\
\hline La mayor parte del día solo/a & 372 & 28,3 \\
\hline $\begin{array}{l}\text { La mayor parte del día } \\
\text { acompañado/a }\end{array}$ & 912 & 69,5 \\
\hline Sin datos & 29 & 2,2 \\
\hline Total & $\mathbf{1 . 3 1 3}$ & $\mathbf{1 0 0 , 0}$ \\
\hline
\end{tabular}

Fuente: Elaboración propia.
3.4. Uso y demanda de servicios y prestaciones sociales

La práctica totalidad de personas en situación de exclusión residencial que han participado en el estudio $(98,3 \%)$ han utilizado algún servicio social o recibido alguna prestación en los últimos tres meses, en el mismo municipio donde fueron entrevistadas, si bien hay un $0,2 \%$ que no lo han hecho (el 1,4\% restante no contestó a la pregunta). Este dato es coherente con el hecho de que el $88,9 \%$ de las personas que participaron en la encuesta fueran entrevistadas en alguno de los recursos existentes, del que eran usuarias en ese momento. Además, se ha podido determinar que, en los tres meses anteriores a la encuesta, cada persona utilizó, de media, casi tres recursos $(3,1$, exactamente).

El recurso más utilizado en los últimos tres meses es, de lejos, el alojamiento, del que se han beneficiado tres de cada cuatro personas sin hogar $(75,1 \%)$. En torno a un tercio han hecho uso de los servicios sociales de base (35\%) y los centros de día (32,2\%), y alrededor de la cuarta parte, de los comedores sociales $(26,5 \%)$ y los talleres de inserción laboral $(24,8 \%)$. Les siguen los servicios de urgencias sociales (19,1\%), la ayuda económica (18,8\%), los centros de inmigrantes (18,4\%), los servicios de higiene o limpieza (18,1\%), los centros de desintoxicación $(10,6 \%)$ y los educadores de calle (9,6\%).

Tabla 19. Distribución de los servicios sociales utilizados por las personas en situación de exclusión residencial grave en los últimos tres meses (respuesta múltiple)

\begin{tabular}{l|c|c}
\cline { 2 - 3 } & Abs. & \% sobre el total \\
\hline Alojamiento & 1.221 & 93,0 \\
\hline Servicios sociales de base & 459 & 35,0 \\
\hline Centro de día & 423 & 32,2 \\
\hline Comedor social & 348 & 26,5 \\
\hline Talleres de inserción laboral & 326 & 24,8 \\
\hline Urgencias sociales & 251 & 19,1 \\
\hline Ayuda económica & 246 & 18,7 \\
\hline Centro de inmigrantes & 242 & 18,4 \\
\hline Servicio higiene/limpieza & 236 & 18,0 \\
\hline Centro de desintoxicación & 139 & 10,6 \\
\hline Educadores/as de calle & 125 & 9,5 \\
\hline
\end{tabular}

Fuente: Elaboración propia.

Una de cada cuatro personas sin hogar encuestadas $(25,1 \%)$ declara que ha solicitado alguna prestación o servicio y que éste le ha sido denegado. Dos tercios $(66,6 \%)$, por el contrario, no se han visto en esa circunstancia. 
Tabla 20. Distribución de las personas en situación de exclusión residencial grave que han solicitado algún servicio o prestación sin haberlo obtenido

\begin{tabular}{l|c|c}
\cline { 2 - 3 } & Abs. & Distribución vertical (\%) \\
\hline Sí & 329 & 25,1 \\
\hline No & 874 & 66,6 \\
\hline Sin datos & 110 & 8,4 \\
\hline Total & 1.313 & $\mathbf{1 0 0 , 0}$ \\
\hline
\end{tabular}

Fuente: Elaboración propia.

Entre los servicios o prestaciones denegadas, figuran, en los primeros lugares, las ayudas económicas en general ( $27,9 \%)$, la renta de garantía de ingresos $(21,4 \%)$ y algún recurso de alojamiento $(15,8 \%)$. Otros recursos que se han denegado con relativa frecuencia son el acceso a Lanbide y los servicios de empleo (7,1\%), el comedor social (5,3\%), el o la trabajadora social (3,7\%) y el servicio de urgencias sociales (3,1\%). Hay que tener en cuenta, en todo caso, que estas cifras representan la valoración que las personas encuestadas hacen de su experiencia, y no necesariamente un registro fehaciente de las solicitudes de prestaciones efectivamente solicitadas y denegadas.

Tabla 21. Distribución de las personas en situación de exclusión residencial grave que han solicitado algún servicio o prestación sin haberlo obtenido, por tipo de recurso denegado

\begin{tabular}{|c|c|c|c|c|}
\hline & Abs. & $\begin{array}{c}\text { \% sobre las } \\
\text { personas } \\
\text { a las que } \\
\text { se les ha } \\
\text { denegado }\end{array}$ & $\begin{array}{c}\% \\
\text { sobre } \\
\text { el } \\
\text { total }\end{array}$ & $\begin{array}{l}\text { Distribución } \\
\text { vertical (\%) }\end{array}$ \\
\hline Ayuda económica & 90 & 27,4 & 6,9 & 27,9 \\
\hline RGI & 69 & 21,0 & 5,3 & 21,4 \\
\hline Alojamiento & 51 & 15,5 & 3,9 & 15,8 \\
\hline Otras ayudas & 27 & 8,2 & 2,1 & 8,4 \\
\hline $\begin{array}{l}\text { Lanbide, servicios de } \\
\text { empleo }\end{array}$ & 23 & 7,0 & 1,8 & 7,1 \\
\hline Comedor social & 17 & 5,2 & 1,3 & 5,3 \\
\hline Trabajador/a social & 12 & 3,6 & 0,9 & 3,7 \\
\hline $\begin{array}{l}\text { Servicio de urgencias } \\
\text { sociales }\end{array}$ & 10 & 3,0 & 0,8 & 3,1 \\
\hline $\begin{array}{l}\text { Ayuda para tramitar } \\
\text { papeles }\end{array}$ & 7 & 2,1 & 0,5 & 2,2 \\
\hline $\begin{array}{l}\text { Más noches de } \\
\text { alojamiento }\end{array}$ & 5 & 1,5 & 0,4 & 1,5 \\
\hline Cursos de formación & 4 & 1,2 & 0,3 & 1,2 \\
\hline $\begin{array}{l}\text { Centro de } \\
\text { desintoxicación } \\
\text { de drogas }\end{array}$ & 3 & 0,9 & 0,2 & 0,9 \\
\hline $\begin{array}{l}\text { Servicio de higiene o } \\
\text { limpieza }\end{array}$ & 2 & 0,6 & 0,2 & 0,6 \\
\hline Centro de día & 1 & 0,3 & 0,1 & 0,3 \\
\hline $\begin{array}{l}\text { Educadores/as } \\
\text { de calle }\end{array}$ & 1 & 0,3 & 0,1 & 0,3 \\
\hline $\begin{array}{l}\text { Comedor social } \\
\text { (no bocadillos) }\end{array}$ & 1 & 0,3 & 0,1 & 0,3 \\
\hline Total & 323 & - & 24,6 & 100,0 \\
\hline
\end{tabular}

Fuente: Elaboración propia.
En lo que se refiere al alojamiento, las personas que han mencionado los motivos de denegación (18 sobre los 56 casos) apuntan cuatro razones: falta de plazas (6 personas), limitación de la estancia (6), imposibilidad de acceso a recursos de emancipación (4) y mal comportamiento (2).

\section{El peso de la edad, el sexo, la nacionalidad y la duración del sinhogarismo en las condiciones de vida}

El estudio realizado ha permitido también comparar las condiciones de vida de las personas en situación de exclusión residencial grave desde la perspectiva de sus características individuales y, más concretamente, en función de variables tales como la edad, el sexo, la nacionalidad, el nivel educativo, el territorio de residencia, el lugar de pernocta o el tiempo transcurrido desde la pérdida de un hogar independiente.

El análisis de estas variables indica que cuatro elementos -la edad, el sexo, la nacionalidad y la prolongación del sinhogarismo- juegan un papel crucial a la hora de explicar la situación y condiciones de vida de estas personas.

- En lo que se refiere a la edad, debe señalarse en primer lugar que se trata de un factor claramente relacionado con otros, como, fundamentalmente, la nacionalidad, en la medida en que las personas extranjeras tienden a ser más jóvenes. El impacto de la edad, por tanto, puede estar mediado por otros elementos, como la nacionalidad o la duración del sinhogarismo.

- En cualquier caso, las personas más mayores (con más de 45 años) son predominantemente autóctonas; suelen tener mayores tasas de inactividad; pernoctan en mayor medida en la calle o en centros de acogida nocturna y albergues; perciben en mayor medida prestaciones económicas (y, por tanto, tienen ingresos más elevados); tienen, lógicamente, una trayectoria de sinhogarismo más larga; tienen un peor estado de salud, fuman y beben más; y tienen menos relaciones sociales. Por su parte, los menores de 30 años son predominantemente (en un $90 \%$ ) extranjeros, tienen una menor presencia en la calle o centros de atención primaria, se dedican en mayor medida a actividades formativas, acceden en menor medida a prestaciones económicas, tienen un mejor estado de salud, consumen drogas en menor medida y mantienen en mayor proporción que el resto algún contacto con su familia de origen.

- La nacionalidad resulta también una variable fundamental a la hora de explicar las condiciones de vida de las personas en situación de exclusión residencial grave, identificándose en general la nacionalidad española con una mayor edad y con situaciones más severas de exclusión. De esta manera, si entre las personas de nacionalidad española el sinhogarismo es producto de (o tiene como consecuencia) procesos de exclusión más 
profundos, entre las personas inmigrantes se relaciona con situaciones de exclusión menos acentuadas. Así, por ejemplo, las personas de nacionalidad española acceden en mayor medida a los recursos especializados (distintos de los albergues y centros de acogida); registran tasas de inactividad más elevadas; acceden en mayor medida a la RGI; tienen ingresos mucho más elevados (prácticamente, el doble); tienen una trayectoria de sinhogarismo más larga; achacan en mayor medida su situación a factores personales; tienen peor estado de salud y consumen en mayor medida alcohol, tabaco y drogas ilegales; y tienen una red de relaciones familiares más débil.

- En lo que respecta al sexo, el dato principal se refiere a la menor prevalencia de las situaciones de exclusión residencial grave entre las mujeres, que apenas representa el $17 \%$ de toda la población encuestada. Las mujeres tienden a ser en mayor medida de nacionalidad española, son algo más mayores que los hombres, utilizan en mayor medida servicios especializados (es decir, duermen menos frecuentemente en la calle 0 en albergues y centros de acogida), acceden en mayor medida a la RGI y cuentan con ingresos más elevados, tienen trayectorias más cortas de sinhogarismo, achacan en mayor medida su situación a motivos relacionales, han dormido en menor medida en la calle a lo largo de sus vidas, y han sido víctimas de agresiones sexuales en mucha mayor medida. Tienen además un estado de salud similar al de los hombres, aunque con una mayor prevalencia de los problemas relacionados con la salud mental, consumen drogas en menor medida y tienen una red de relaciones personales algo más amplia que los hombres.

- Finalmente, y como cabía esperar, el tiempo durante el que se prolonga la situación de exclusión residencial se relaciona con unas condiciones de vida más difíciles. Así, el grupo de quienes llevan más de cinco años en esta situación -en el que predominan los hombres mayores y de nacionalidad española- está compuesto en mayor medida por personas inactivas, accede en mayor medida a la RGI, tiene ingresos más elevados, ha dormido en la calle en mayor medida, ha sido víctima de delitos y amenazas en mayor medida, tiene un peor estado de salud y una mayor prevalencia de enfermedades crónicas o graves, consume drogas (especialmente ilegales) en mayor medida, y mantiene menos contactos familiares y sociales.
Aunque existen, se han encontrado menos diferencias, por el contrario, en lo que se refiere al lugar de pernocta durante la noche del recuento y al territorio de residencia.

- En lo que se refiere a lugar de pernocta, cabe señalar que las personas localizadas en la calle y las localizadas en albergues y centros de acogida tienen características similares. Con todo, las personas localizadas en la calle destacan por una tasa de empadronamiento mucho más baja, una actividad laboral más frecuente, menor acceso a la RGI y a otras prestaciones económicas, y mayor consumo de drogas.

- Desde el punto de vista territorial, no se observan datos que permitan hablar de perfiles homogéneos y diferentes en cada territorio, lo que, lógicamente, no excluye la existencia de ciertas diferencias. Así, Álava se caracteriza por un mayor peso de la población femenina, autóctona y mayor de 45 años; una mayor proporción de personas alojadas en albergues y centros de acogida; un mayor peso de las personas con estudios universitarios; una tasa de actividad más baja; una cobertura de la RGI también más baja; y por una proporción mayor de personas que nunca han dormido en la calle.

Por su parte, las personas localizadas en Bizkaia se definen por ser en mayor medida hombres, pernoctar en la calle en mayor proporción, no haberse empadronado en mayor proporción, beneficiarse de una cobertura de la RGI mucho más elevada (del 23,7\%, frente al 7,1\% de Álava y al 15,4\% de Gipuzkoa), y por contar con unos ingresos medios más elevados y un estado de salud algo peor. Finalmente, Gipuzkoa se caracteriza por un mayor peso de la población extranjera, una proporción mayor de personas extranjeras con permiso de residencia y de personas empadronadas, un menor porcentaje de personas sin estudios, una menor proporción de personas inactivas, y por un porcentaje muy elevado de personas que sólo perciben ingresos de fuentes privadas. También se define por tener un número más elevado de personas que señalan tener una situación de salud buena 0 muy buena, una menor prevalencia de enfermedades y una menor proporción de personas que pasan la mayor parte del tiempo solas o que no mantienen ningún tipo de contacto familiar. 
(2008): "Ley 12/2008, de Servicios Sociales del País Vasco", Boletín Oficial del País Vasco, $\mathrm{n}-246,24$-122008, págs. 31.840-31.924 [<http://www. euskadi.net/bopv2/datos/2008/12/0807143a. pdf〉].

ANGULO, M. C. et al. (2011): Informe Nessun Dorma. Recuento de personas sin hogar en la ciudad de Sevilla 2011, Sevilla, Asociación Pro-Derechos Humanos de Andalucía.

AVRAMOV, D. (1996): The Invisible Hand of the Housing Market: A Study of Effects of Changes in the Housing Market on Homelessness in the European Union, Bruselas, Feantsa.

- (1995): Homelessness in the European Union: Social and Legal Context of Housing Exclusion in the 1990 s. Fourth Research Report of the European Observatory on Homelessness, Bruselas, Feantsa.

CABRERA, P. J. (2010): Estudio sobre las personas sin techo en Zaragoza, Zaragoza, Cruz Roja Española.

CABRERA, P. J. et al. (2008): ¿Quién duerme en la calle? Una investigación social y ciudadana sobre las personas sin techo, serie: Herramientas para la Inclusión, nº 4, Barcelona, Caixa Catalunya.

CHITYIL, B. et al. (2010): “Gender perspectives on homelessness", en Homeless in Europe, no spring.

CRUZ TERÁN, J. (dir.) (2006): Vivir en la calle. Informe especial sobre la situación de las personas sin techo en Andalucía, Sevilla, Defensor del Pueblo Andaluz.

DELOITTE (2010): Análisis de situación de las personas en situación de exclusión residencial grave, Bilbao, Grupo de Trabajo Onartu; Bilbao, Ayuntamiento de Bilbao.

EUSTAT (2012): Encuesta sobre las Personas sin Hogar 2012, Vitoria-Gasteiz, Eustat.

FEANTSA (2005): ETHOS - European Typology of Homelessness and Housing Exclusion, Bruselas, European Federation of National Organisations Working with the Homeless [<http://www.feantsa.org/spip. php?article120\&lang=en>].
GOBIERNO VASCO, Departamento de Empleo y Asuntos Sociales (2012): Encuesta de pobreza y desigualdades sociales 2012. Principales resultados, Vitoria-Gasteiz, Eusko JaurlaritzaGobierno Vasco.

GOBIERNO VASCO, Departamento de Salud (2012): Euskadi y drogas 2012, Vitoria-Gasteiz, Departamento de Salud del Gobierno Vasco.

MINISTERIO DE SANIDAD, POLIITICA SOCIAL E IGUALDAD (2011): Configuración de una red local de atención a personas sin hogar integrada en el sistema público de servicios sociales. 100 Argumentos y propuestas, serie: Informes, Estudios e Investigación, Madrid, Ministerio de Sanidad, Política Social e Igualdad.

MUÑOZ, M. et al. (dirs.) (2012): Operación de recuento nocturno de personas viviendo sin techo en las calles de Madrid. 13 de diciembre de 2012. Principales resultados, Madrid, Ayuntamiento de Madrid.

NEWBURN, T.; y ROCK, P. (2005): Living in Fear: Violence and Victimisation in the Lives of Single Homeless People, Londres, LSE Mannheim Center of Criminology.

SALES, A. (2012): La situació de les persones sense llar a Barcelona el 8 de novembre de 2011 i l'evolució dels serveis residencials, Barcelona, Xarxa d'Atenció a Persones sense Llar de Barcelona.

SIIS CENTRO DE DOCUMENTACIÓN Y ESTUDIOS (2013): Estudio sobre la situación de las personas en situación de exclusión residencial grave en la CAPV, Vitoria-Gasteiz, Eusko JaurlaritzaGobierno Vasco [<http://www.siis.net/es/verdetalle.php?ref=211784'].

- (2011): Kale Gorrian. Estudio sobre la situación de las personas sin hogar en Donostia-San Sebastián / Kale Gorrian. Donostian etxerik gabeko pertsonen egoerari buruzko azterlana, Donostia-San Sebastián, Ayuntamiento de Donostia-San Sebastián; Donostia-San Sebastián, Kale Gorrian [<http://www.siis.net/ es/ver-detalle. php? $r e f=201365$ '].

VALEDOR DO POBO (2010): Las personas sin hogar en Galicia, Santiago de Compostela, Valedor do Pobo. 